during the study. Ten (38\%) of the subjects who received the control formula and $3(10 \%)$ of those who received the supplemented formula shed rotavirus at some time during the study. The researchers concluded that a decrease in rotavirus shedding may lead to less environmental exposure, which may contribute to a reduced risk of nosocomial infection in infants at risk of gastroenteritis.

FROM: Saavedra JM, Bauman NA, Oung I, et al. Feeding of Bafidobacterium bifidum and Streptococcus thermophilus to infants in hospital for prevention of diarrhea and shedding of rotavirus. Lancet October 15, 1994;344:1046-1049.

\section{New Research Suggests Herpes Virus May Cause Kaposi's Sarcoma}

Dr. Patrick Moore and colleagues from Columbia University recently presented research findings that suggest herpes virus may be the cause of Kaposi's sarcoma (KS). These findings were presented during a symposium at the Second National Conference on Human Retroviruses held on January 31, 1995, in Washington, DC. The virus has been tentatively named Kaposi Sarcoma-Associated Herpes Virus (KSHS).

The virus is being grown in tissue culture in the laboratory and mapping of the virus's basic genetic structure has identified it as the largest human herpes virus. Dr. Moore found evidence of the KSHS in 20 out of 21 tissue samples from individuals with KS, compared to only 1 of 21 samples of individuals without KS.

Dr. Steven Miles, from the University of California, Los Angeles Medical School, and co-chair of the sesson, said that Dr. Moore's findings represent a "major breakthrough in KS and will change the scope of research."

In the same session, Dr. David Cooper, from the University of New South Wales, Australia, presented the results of a clinical trial to treat patients with $\mathrm{KS}$ using an experimental drug made by Abbott Laboratories, ABT 538, a protease inhibitor, which resulted in complete or partial regression of disease.

FROM: Roundtable Symposium: Controversies in AIDS Malignancies. Presented at the Second National Conference on Human Retroviruses and Related Infections. January 29 Feb ruary 1.1995; Washington DC.

\section{FDA Approves Oral HIV Test}

The US Food and Drug Administration (FDA) has approved the first US HIV test system using oral fluid samples instead of blood. FDA has approved the product for collecting specimens of oral fluid and as an ELISA antibody screening test specifically licensed for testing oral fluid samples. The collection device, called OraSure, includes a specially treated cotton pad on a stick and a preservative solution in a plastic container in which to store the specimen until the sample is tested by a qualified laboratory using the Oral Fluid Vironostika HIV-1 Microelisa System.
The FDA has approved this HIV test system with a number of restrictions, including distribution only through physicians, collection done only by properly trained individuals, and restriction from home use. There is also a requirement for providing a subject information sheet to individuals being tested that includes information on the reduced accuracy of testing oral fluids compared with testing blood, the lack of a confirmatory test for use with oral fluid samples, and, if the test is positive, the need to have blood samples drawn and tested to verify HIV status.

The OraSure HIV-1 specimen collection device is manufactured by Epitope Corporation of Beaver-ton, Oregon, and the Oral Fluid Vironostika HIV-l Microelisa System approved to test the specimens is manufactured by Organon Teknika Corporation of Durham, North Carolina.

\section{HIV-Infected Women Have Increased Risk of Death, Not Disease Progression}

HIV-infected women are one third more likely to die without an AIDS-defining condition than are HIV-infected men, according to a study from the National Institute of Allergy and Infectious Disease (NIAID). The investigators could not identify why women had a greater risk of relatively early death but suggest that important factors may involve poorer access to or use of healthcare resources among HIV-infected women as compared to men, domestic violence, and lack of social supports for women.

A total of 768 women and 3,779 men were followed for 15 months in this study, the largest prospective analysis comparing survival and disease progression between HIV infected women and men, while controlling for predictors of disease. Among the surviving HIV-infected people in the study, no gender differences occurred in the risk of subsequently developing an AIDS-associated condition. For both men and women, the most commonly occurring AIDS associated condition was Pneumocystis carinii pneumonia.

AIDS among women in the United States now represents nearly $13 \%$ of all cases, a more than 20-fold increase since 1981. According to the CDC, the disease is the leading cause of death for women in New York City age 25-44 and among the top five leading causes of death for US women of these same ages.

FROM: Melnick SL, Sherer R, Louis TA, et. al. Survival and disease progression according to gender of patients with HIV Infection JAMA 1995;272 (24):1915-1921.

Additional news items in this issue: OSHA Expands Focused Inspections in Face of Limited Resources (page 159), Difference in Antibody Response Rate Between Engerix-B and Recombivax-HB Has No Public Health Significance (page 165). 